Nuclear Explosion Monitoring Research and Engineering (NEMR\&E) Program Quarterly Report - BAA06-37, Contract No. DE-FC52-06NA27323

\title{
Mechanically Cooled Large-Volume Germanium Detector Systems for Nuclear Explosion Monitoring
}

Ethan Hull (PI)

PHDs Co.

777 Emory Valley Road

Suite B

Oak Ridge, TN 37830

(865) 481-3725

Reporting period: July-September 2006

\section{Description of activities conducted this Reporting Period:}

1. In early August, the principal investigator (Ethan Hull) traveled to PNNL for a meeting with the product integrator (Ted Bowyer) and collaborators (Harry Miley et. Al.). The visit was extremely productive. Dr. Hull presented the plan of attack for development of better detectors for the RASA system. We toured and photographed the RASA system at PNNL that will be used for the demonstration of the improved detector system. We also obtained mechanical drawing of the entire RASA system. This enabled us to modify our prototype design to fit easily into the RASA and provide a real functioning system.

2. In September, Dr. Hull attended the SRR in Orlando, FL. Although the dominant attendance of the conference is related to seismic research, Dr. Hull found himself inundated with interest in the RASA detectors we seek to develop under this BAA. Extensive discussions were held with DOE officials (Leslie Casey, Joe Wehlburg), University researchers (Steven Biegalski), AFTAC scientists (George Rybicki, David Merker, et al.), contract administrator scientists (Joseph Romero, Jeffery Rivas), and members of the PNNL team (Justin Mclntyre et al.). These discussions served as additional inspiration for the immediate need for better, more reliable detector system for nuclear explosion monitoring. As a result of these discussions we have decided to attempt to expedite the fabrication of a first prototype that can be useful to alleviate the problems. This is discussed further below. We presented two papers at the conference.

3. Work on the Vacuum-RGA system has continued. We found that the original RGA system purchased (Ametek 100-AMU LC100M Residual Gas Analyzer) was faulty. We had many discussions with Ametek in attempt to rectify the problems. After much iteration it became clear that this system was not suitable for our application. We returned it and purchased a similar system from MKS (MKS eVISION 100F Tungsten). The MKS unit is fully contained immediately at the location of the RGA on the vacuum system. This eliminates much of the cabling that was problematic with the Ametek 
unit. We now achieve extremely consistent and accurate ultra-high vacuum performance and gas species identification. We have ordered two vacuum cavities for residual out-gassing measurements. We have also ordered two more Varian metal internal sealing vacuum valves to allow gas accumulation over time. We regularly achieve low $10^{-9}$ torr pressures in the pumping station with hydrogen as the dominant gas species. Figure 1 shows a photograph of the new system operating at our Oak Ridge, TN facility.

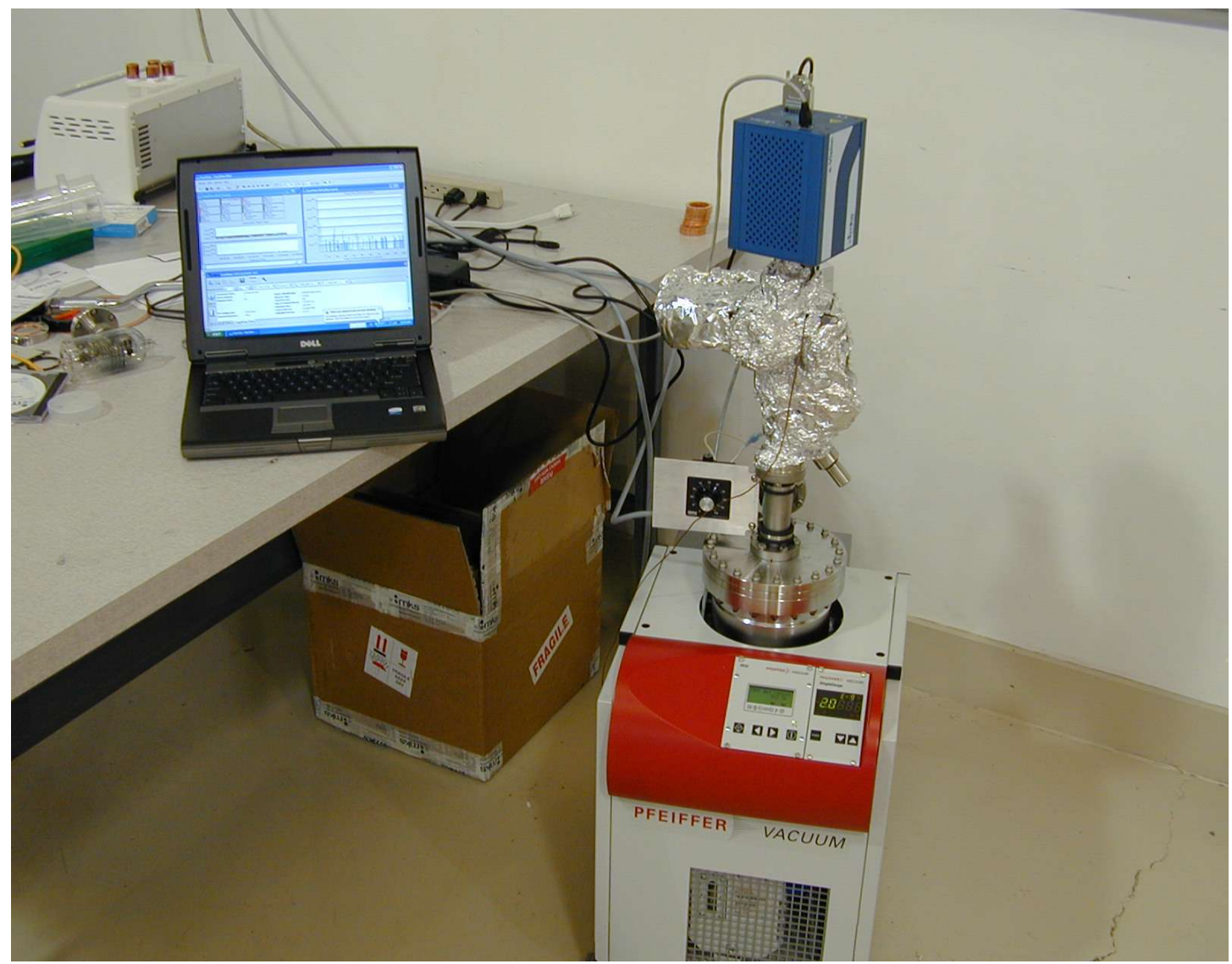

Figure 1 The photograph shows the turbo molecular pump in with the new MKS RGA operating over the pump. A histogram of the RGA output can be seen on the laptop computer on the bench to the left. The system achieves base vacuum in the low $\times 10-9$ Torr region. This should be more than sufficient to measure the accumulated gases affecting the operation of mechanically cooled germanium detectors.

4. In the previous report we discussed the beginnings of the first prototype design we called MCO-a. As a result of the trip to PNNL and the SRR we decided to change the name of the first prototype to RASA 1. RASA 1 is specifically designed to fit into and operate with a working RASA. We are going to fabricate a 7-cm diameter $\times 7$-cm long coaxial germanium detector to function in RASA 1. This is a result of the immediate need for such a system as expressed to us by the AFTAC users at Patrick Air Force base. RASA 1 has been entirely designed. The parts for RASA 1 have been 
ordered. The parts include the machined cryostat vacuum parts, electronic assembly parts, exterior assembly parts, fan, mechanical cooler, and germanium detector crystal. We have placed an emphasis on the loading of the coaxial detector to assure a rugged assembly. The detector is held in a specially designed capsule containing wave springs that can hold the detector with a force equivalent as high as $100 \mathrm{Gs}$ if desired. We have also included provisions to shield high voltage components from the signal gate lead to prevent microphonic noise. We are awaiting delivery of these parts. The figure below shows the assembly of RASA 1.

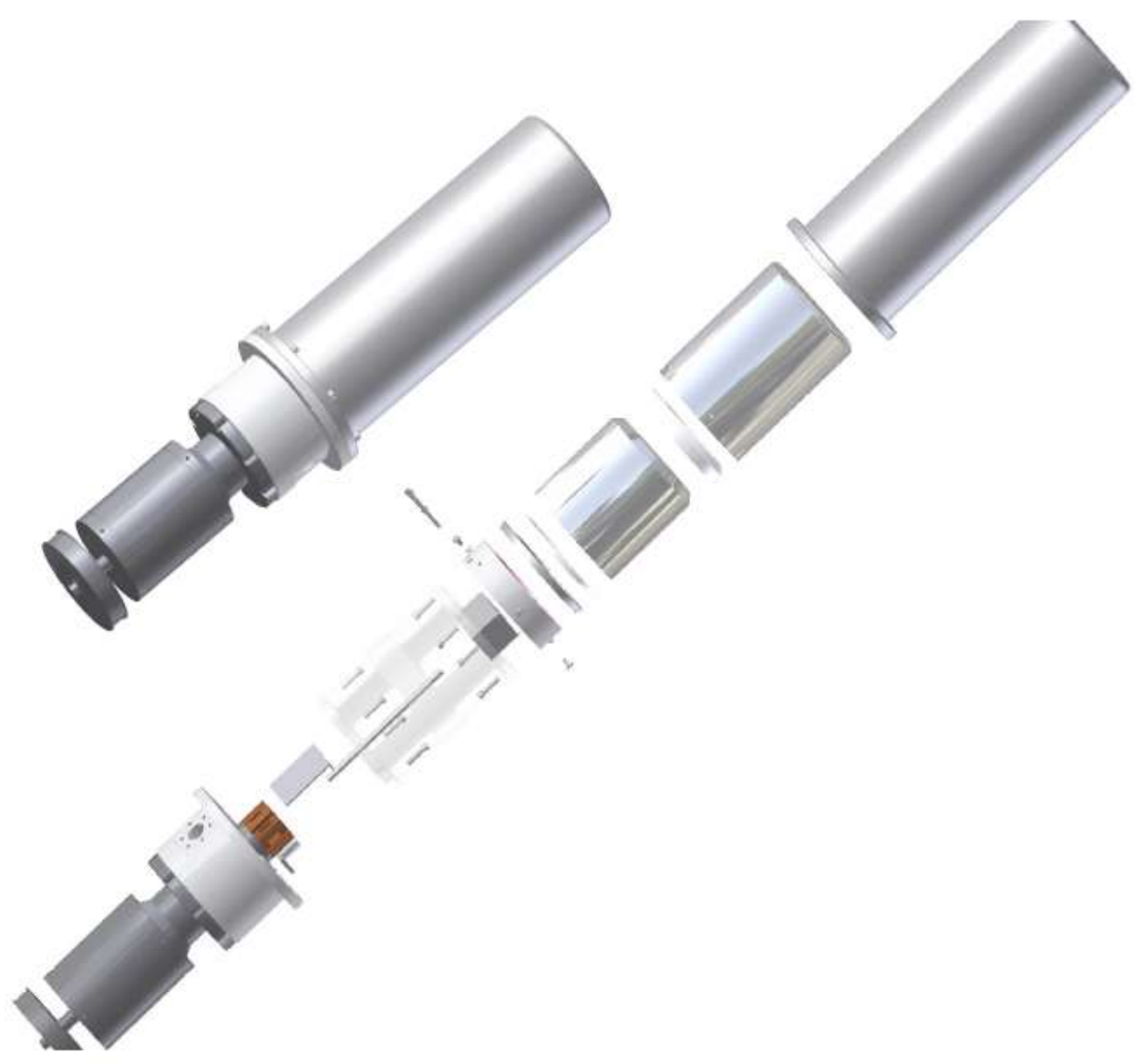

Figure 2 The assembly drawing shows the final design of first prototype mechanically cooled system to be built, RASA 1 . The system is large enough to accomodate the largest germanium detector available; a 10-cm diameter, 10-cm long detector.

\section{Progress/Deliverables Required By this Reporting periods Per Contract:}

The highlighted items $a, b, c, f$, and $\mathrm{k}$ required and received attention during these months of the Phase 1 (year 1) of the program. 


\section{PHASE 1 (12 months)}

a. Build Vacuum-RGA system (months 1-2) Acquire equipment including turbo-molecular vacuum system, RGA, and computers to operate RGA and cooler.

b. Establish operation parameters for sensitive accumulated gas measurements (month 2)

c. Design and build first test cryostat (MC0-a) using Cryotel CT cooler from Sunpower with brass detector dummy (months 1-3).

d. Measure cooler performance as a function of time and vacuum quality (month 3).

e. Make measurements of accumulated gases in test cryostat (months 4-5).

f. Attend SRR.

g. Address predominant accumulated gases (months 6-8).

h. Design and fabricate MC1 (months 8-10).

i. Load planar detector into MC1 and evaluate microphonics (month 11).

j. Load $50 \%$ coaxial detector into MC1 at Ortec and deliver to PNNL (month 12).

k. Meetings with Product Integrator.

\section{Reason for Differences in Cooperative Agreement Statement of Objectives/Deliverables}

No differences, everything is on schedule. 